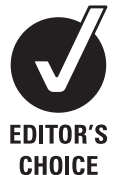

Southend University Hospital NHS Trust, Southend on Sea, UK

${ }^{2}$ Centre for Applied Medical Statistics, University of Cambridge, Institute of Public Health, Cambridge, UK

\section{Correspondence to}

Dr P Guyler, Department of Stroke Medicine, Southend Hospital NHS Trust, Prittlewell Chase, Southend on Sea SSOORY, UK;

paul.guyler@southend.nhs.uk

Received 8 July 2010

Revised 17 December 2010

Accepted 21 December 2010

Published Online First

3 February 2011

\title{
Intravenous thrombolysis in acute ischaemic stroke: a systematic review and meta-analysis to aid decision making in patients over 80 years of age
}

\author{
Pallav Bhatnagar, ${ }_{1}^{1}$ Devesh Sinha, ${ }^{1}$ Richard A Parker, ${ }^{2}$ Paul Guyler, ${ }^{1}$ Anthony 0'Brien ${ }^{1}$
}

\begin{abstract}
Introduction Patients $\geq 80$ years of age are increasingly receiving intravenous thrombolysis for acute ischaemic stroke (AIS) despite lack of firm evidence. This systematic review assesses the safety and efficacy of intravenous thrombolysis with alteplase in $\geq 80$ versus $<80$ year old patients with AIS.
\end{abstract}

Methods The existing literature was systematically analysed for outcome measures of mortality, functional recovery by modified Rankin scale and symptomatic intracranial haemorrhage (SICH) at 3 months following intravenous thrombolysis with alteplase in $<80$ and $\geq 80$ year old patients with AIS. Statistical tests were performed for heterogeneity and publication bias. A detailed sensitivity analysis was performed and Forest plot was constructed for each of the outcome measures. Results 13 studies were identified. The overall OR was 2.77 (95\% Cl 2.25 to 3.40) for death, 0.49 (95\% Cl 0.40 to $0.61)$ for achieving a favourable outcome and $1.31(95 \% \mathrm{Cl}$ 0.93 to 1.84 ) for SICH in $\geq 80$ year old patients compared with those $<80$ years old. The total number of events contributing to the estimates of effect for each outcome was: death 199, favourable outcome 141 and SICH 49. Conclusion Patients $\geq 80$ years of age appear to have a lower probability of gaining a favourable outcome and a higher mortality rate compared with patients $<80$ years old; however, the rate of SICH was not significantly increased. This supports recruitment of patients aged $\geq 80$ years into ongoing trials comparing thrombolysis with controls. For patients who refuse or cannot be randomised, it provides information on risks and benefits of using alteplase off-licence.

\section{INTRODUCTION}

The safety and efficacy of intravenous thrombolysis with alteplase, a reverse transcriptase plasminogen activator (tPA), licensed for use in a select population with acute ischaemic stroke (AIS), is well established. ${ }^{1}$ In the UK, about 30000 people over 80 years of age have AIS each year but they do not receive tPA because of their age and concern of causing symptomatic haemorrhage. The elderly (over 80 years) population have previously been subject to discrimination in trials of thrombolytic therapies. There have been attempts to justify their inclusion on the basis of data from non-randomised monitoring cohorts. Thrombolysis in the above 80 year old group is increasing and the SITS-MOST (Safe Implementation of Thrombolysis and Monitoring Study) database currently documents approximately 4000 of such patients in 450 centres across 33 countries. ${ }^{2}$ The question that clinicians are increasingly facing is: what is the safety and efficacy of intravenous thrombolytic therapy in the elderly population? The ongoing International Stroke Trial-3 (IST-3) ${ }^{3}$ is likely to present its findings in April 2012 but until then, stroke physicians need some information to help patients unable or unwilling to be part of thrombolysis trials to make an informed choice regarding off-licence use of alteplase. To try and help until such trials report, we have performed a systematic review of the current literature. The last systematic review published in 2006 by Engelter ${ }^{4}$ had a limited look at outcomes of intravenous thrombolysis in stroke patients of $\geq 80$ versus $<80$ years of age. Since then more evidence has become available that we have analysed in this paper to reach a clinical bottom line. We believe this analysis is timely and justifies the continuation of trials that seek to obtain information on the balance of risk and benefit to populations presently excluded from treatment.

\section{METHODS}

\section{Methodology of systematic review}

The existing literature was systematically searched by two independent authors (PB and DS) using National Information Resources facility for Medline (1950 onward), Embase (1980 onwards) and CINAHL (1981 onwards) databases until September 2010 for various terms related to 'acute isch(a)emic stroke' and 'thrombolysis' using thesaurus mapping and truncation as appropriate to maximise the scope of search. The following PICOS strategy was used to search the various databases: population: elderly patients $\geq 80$ years old thrombolysed for AIS; intervention: thrombolysis with tPA (alteplase); comparison: patients $<80$ years old thrombolysed for AIS; outcomes: death, functional recovery by modified Rankin scale $(\mathrm{mRS})$ and symptomatic intracranial haemorrhage (SICH) at 3 months; study design: comparative observational cohort studies; non-comparative case series, isolated case reports, reviews and comments were excluded. The leading journals and bibliographies of selected articles were hand searched. The studies satisfying inclusion and exclusion criteria were then individually appraised by $\mathrm{PB}$ and DS. Any disagreement was resolved by mutual discussion involving PG.

\section{Statistical analysis}

For each outcome of interest $(\mathrm{SICH}, \mathrm{mRS}$ and mortality at 3 months), a separate fixed effects meta-analysis was performed utilising the MantelHaenszel method to calculate an overall summary measure. $^{5}$ 
For all fixed effects meta-analyses, it was assumed that the true effect sizes were the same for all studies and any difference observed was simply due to sampling variation. A $\chi^{2}$ test of heterogeneity ( $\mathrm{Q}$ ) was applied in each case to assess the fixed effects assumption. A $10 \%$ significance level was used for the test of heterogeneity.

For each of the outcomes of interest, forest plots were produced to show the effect sizes (ORs) for each of the relevant studies with an overall summary estimate (OR) generated from the meta-analysis. Corresponding 95\% CIs were shown alongside the ORs. Publication bias was investigated for each meta-analysis using funnel plots with SE on the y axis and effect size on the $\mathrm{x}$ axis.

In meta-analyses of observational studies, biased effect sizes for individual studies are an unfortunate possibility and therefore any biases in individual studies are likely to propagate into the overall summary measure. Therefore, Egger et al suggest that exploring the possible sources of heterogeneity between observational study results should be an important feature of metaanalyses of observational studies. ${ }^{6}$ We believe a non-significant test result of heterogeneity is not enough to have confidence in the overall summary measure as these tests often lack power so low levels of heterogeneity may still exist in practice. In addition, a hypothesis test provides no substitute for a thorough comparison of the studies. Therefore, with this in mind, in our systematic review we carefully assessed heterogeneity between studies and also tested the stability of the overall summary measure by performing a thorough sensitivity analysis for each meta-analysis.

The statistics software used was the 'Metafor' package ${ }^{7}$ in the $\mathrm{R}$ statistics software. ${ }^{8}$

\section{RESULTS}

\section{Results of systematic review}

The systematic search returned 13 comparative cohort observational studies that appeared to answer the question, making it the most comprehensive review to date. Two papers ${ }^{9} 10$ used similar methodology but calculated inhospital death and/or disability at discharge rather than at 3 months; hence only data on $\mathrm{SICH}$ were taken from them to maintain uniformity of analysis. One study $^{11}$ was alleged to have been included in a larger national multicentre study ${ }^{12}$ in a previous review. ${ }^{4}$ We included this study and performed a sensitivity analysis to investigate the effect of this study on the overall summary estimates.

All studies had well defined methodology and collected data prospectively. Ringleb ${ }^{13}$ and Mouradian ${ }^{11}$ followed their local and national protocols. Subsequent sensitivity analysis, excluding these studies, revealed very little change in the overall summary ORs. The majority of authors attempted to compare most of the known, potentially confounding, baseline characteristics of the two populations, except Ringleb ${ }^{13}$ and Berrouschot ${ }^{14}$ who compared only a few characteristics such as baseline National Institutes of Health Stroke Scale, sex and time to treatment. Four authors ${ }^{12} 131516 \mathrm{did}$ not mention if they included consecutive thrombolysed patients but it appears from their results that they did. Seven authors (table 1) mentioned protocol violations and among them Tanne ${ }^{10}$ had protocol violations significantly different between the two groups. Losses to follow-up were accounted for in two studies at 3 months. It was not considered relevant for Tanne ${ }^{10}$ and $\mathrm{Chen}^{9}$ as the outcome measures of death and disability were calculated at discharge. The individual characteristics of the studies are tabulated in table 1 and details of each study, including results, are mentioned in table 2. Uyttenboogaart ${ }^{17}$ performed multivariate analysis to adjust for possible confounders and eight other studies ${ }^{10-12} 14-16181921$ performed logistic regression analysis to adjust for the differences in important baseline characteristics and to identify predictors of favourable and/or poor outcomes. Our analysis showed that there was little evidence to suggest publication bias in funnel plots for the three outcomes (figure 1).

\section{Death at 3 months}

Mortality at 3 months was reported by 11 studies. Results by Mouradian ${ }^{11}$ were excluded from the meta-analysis as they reported only stroke related deaths at 3 months. The combined (fixed) OR, as represented by a diamond at the bottom of the plot in figure 2, was calculated to be 2.77 (95\% CI 2.25 to 3.40) from 10 studies, suggesting an increased likelihood of death at 3 months in the thrombolysed over 80 year old group. Including Mouradian ${ }^{11}$ in the analysis did not change the results significantly (OR 2.80 (95\% CI 2.29 to 3.43 )).

The test for heterogeneity gave a $\mathrm{Q}$ score of 11.98 on 9 degrees of freedom. This corresponds to a $p$ value of 0.21 , which is non-significant at the $10 \%$ level. Therefore, there was insufficient evidence of any heterogeneity between the studies.

Sensitivity analysis performed to adjust for minor variations among the studies showed little change in the overall summary $\mathrm{OR}$ and our conclusions remained the same in each case. When the Gomez-Choco ${ }^{18}$ paper was removed however, the overall summary estimate of the OR increased by 0.15 , but our overall conclusions did not change.

Table 1 Characteristics of the appraised studies

\begin{tabular}{|c|c|c|c|c|c|}
\hline Studies & Centre & Prospective data collection & Protocol & Protocol violations & Losses to follow-up \\
\hline Toni $^{15}$ & Multi, Italy & $\sqrt{ }$ & NINDS & Described $(p=0.59)$ & Not described \\
\hline Uyttenboogaart $^{17}$ & Single, Netherlands & $\sqrt{ }$ & NINDS & Not described & Not described \\
\hline Gómez-Choco ${ }^{18}$ & Single, Spain & $\sqrt{ }$ & NINDS & Not described & Not described \\
\hline Meseguer $^{19}$ & Single, France & $\sqrt{ }$ & NINDS & Described $(p=1.0)$ & Described \\
\hline Ringleb $^{13}$ & Single, Germany & $\sqrt{ }$ & Local & Not described & Not described \\
\hline Mouradian ${ }^{11}$ & Single, Canada & $\sqrt{ }$ & National & Not described & Not described \\
\hline Berrouschot $^{14}$ & Multi, Germany & $\sqrt{ }$ & NINDS & Described $(p=0.67)$ & Not described \\
\hline Engelter $^{16}$ & Multi, Switzerland & $\sqrt{ }$ & NINDS & Described $(p=0.43)$ & Not described \\
\hline Sylaja $^{12}$ & Multi, Canada & $\sqrt{ }$ & NINDS & Described $(p=0.26)$ & Not described \\
\hline Oostenbrugge ${ }^{20}$ & Single, Netherlands & $\sqrt{ }$ & NINDS & Not described & Not described \\
\hline Parnetti ${ }^{21}$ & Single, Italy & $\sqrt{ }$ & NINDS + EUSI & Described (no difference detected) & Described \\
\hline Tanne $^{10}$ & Multi, USA & $\sqrt{ }$ & NINDS & Described $(p=0.03)$ & None \\
\hline Chen $^{9}$ & Single, USA & $\sqrt{ }$ & NINDS & Not described & None \\
\hline
\end{tabular}

EUSI, European Stroke Initiative recommendations for stroke management-update 2003; NINDS, National Institute of Neurological Diseases and Stroke. 
Table 2 Results of individual studies

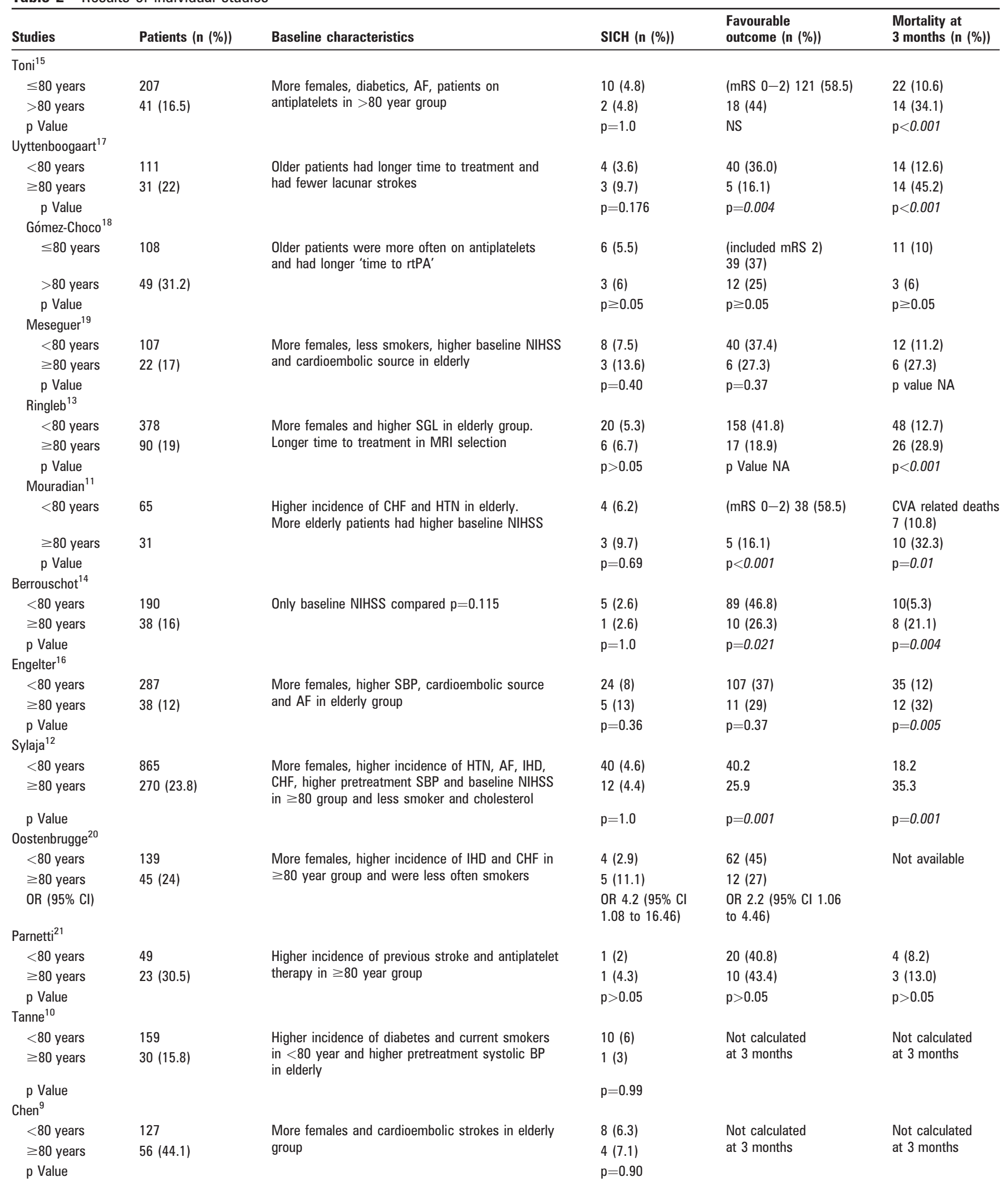

$\mathrm{AF}$, atrial fibrillation; $\mathrm{BP}$, blood pressure; CHF, congestive heart failure; CVA, cardiovascular accident; HTN, hypertension; IHD, ischaemic heart disease; mRS, modified Rankin scale; NIHSS, National Institutes of Health Stroke Scale; NINDS, National Institute of Neurological Diseases and Stroke; rtPA, reverse transcriptase plasminogen activator; SBP, systolic blood pressure; SGL, serum glucose level; SICH, symptomatic intracranial haemorrhage.

Functional outcome at 3 months

Analysis was performed on eight studies that reported favourable outcome as mRS $0-1$. Three studies were not included in the statistical analysis ${ }^{11} 1518$ as they reported favourable outcome as $0-2$. The combined (fixed) OR was calculated to be 0.49 ( $95 \%$ CI 0.40 to 0.61 ), suggesting that patients over 80 years of age are not as likely to achieve a favourable outcome as patients $<80$ years old thrombolysed for AIS. 

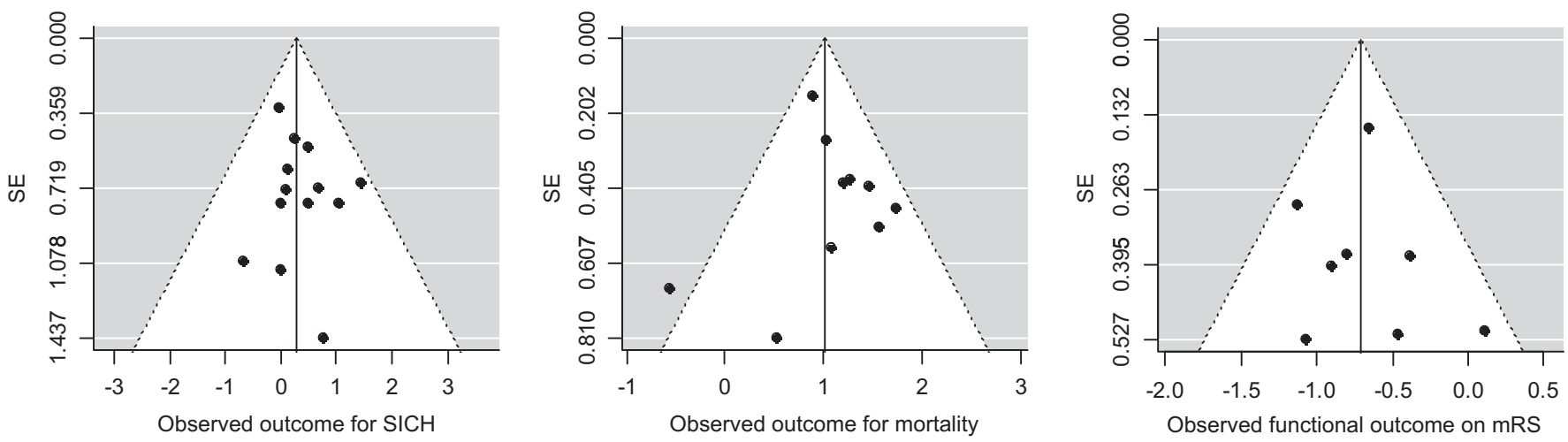

Figure 1 Funnel plots to assess possible publication bias corresponding to meta-analyses performed for symptomatic intracranial haemorrhage (SICH), mortality at 3 months and functional outcome on the modified Rankin scale (mRS).

The test for heterogeneity gave a $\mathrm{Q}$ score of 6.54 on 7 degrees of freedom. This corresponds to a $p$ value of 0.48 , which is non-significant at the $10 \%$ level. Therefore, there was insufficient evidence of any heterogeneity between studies.

A sensitivity analysis was performed which showed very little change in the overall summary $O R$ and our conclusions remained the same.

We considered performing a meta-analysis of favourable outcome as mRS 0-2-ie, we only included the Toni, ${ }^{15}$ GomezChoco $^{18}$ and Mouradian ${ }^{11}$ papers. However, the test for heterogeneity resulted in a $\mathrm{Q}$ statistic of 5.49 on 2 degrees of freedom with a corresponding $p$ value of 0.06 . Therefore, the test was significant at the $10 \%$ level, and it was considered invalid to combine the results.

Incidence of symptomatic intracranial haemorrhage

Studies were noted to have employed two different definitions of SICH. Five studies ${ }^{1013161720}$ used the definition as utilised by the National Institute of Neurological Diseases and Stroke (NINDS) study group ${ }^{22}$ where a haemorrhage was considered symptomatic if it was not seen on a previous CT scan and there had subsequently been a decline in neurological status.

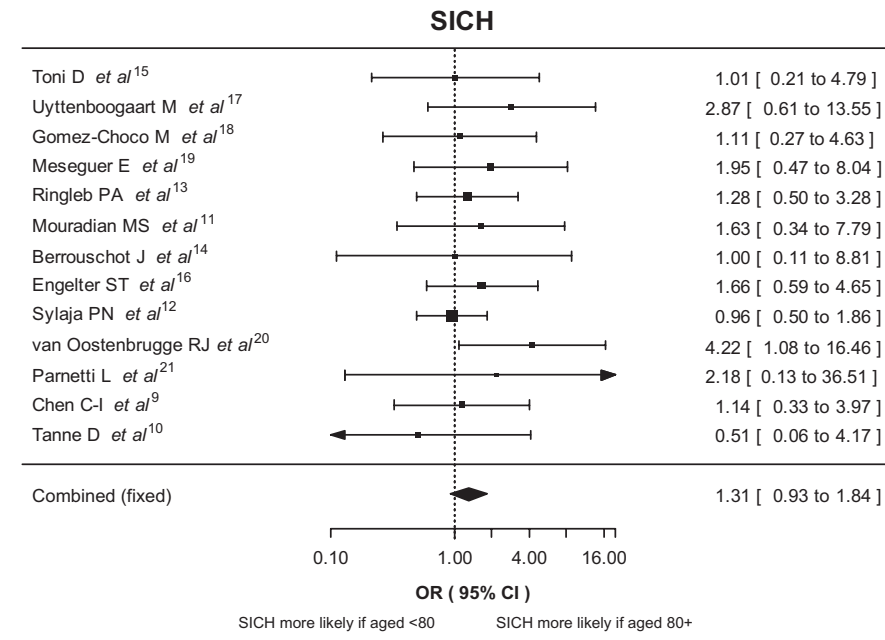
probability of favourable outcome on the modified Rankin scale (mRS) - OR (fixed effects) meta-analysis plots. Right hand side of the plot corresponds to an increased risk for patients $\geq 80$ years old relative to those $<80$ years. 
Eight $^{9} 11121415181921$ used the definition as utilised by the European Cooperative Acute Stroke Study III (ECASS III) trial ${ }^{23}$ where SICH was defined as an intracranial bleed on CT scan with a decrease in the National Institutes of Health Stroke Scale score of 4 or more points.

The combined (fixed) OR of all studies was calculated to be 1.31 (95\% CI 0.93 to 1.84), suggesting no significant difference in the risk of SICH between the two age groups.

The test for heterogeneity gave a $\mathrm{Q}$ score of 6.41 on 12 degrees of freedom. This corresponds to a $p$ value of 0.89 , which is non-significant at the $10 \%$ level. Therefore, there was insufficient evidence of any heterogeneity between studies.

A thorough sensitivity analysis was performed by sequentially removing individual studies that had minor variations and it showed very little change in the overall summary OR and our overall conclusions remained the same.

\section{DISCUSSION}

The question of whether thrombolysis is safe and effective in the elderly population aged over 80 years requires an adequately powered randomised controlled trial (RCT) such as the ongoing IST-3. ${ }^{24}$ Various studies, appraised in this review, attempted to obtain indirect evidence to answer the question by comparing their experience of off-licence use of intravenous thrombolysis in patients over 80 years of age with those in younger patients.

In the absence of an RCT, such comparative cohort observational studies are the next most appropriate research design to be considered, as patients over 80 years old are increasingly being provided thrombolysis as treatment for AIS.

The mean proportion of over 80 year old patients achieving a favourable outcome of $0-1$ was found to be $26.7 \%$ (range $16.1-43.5 \%$; SD 8.1) on pooled analysis of the data from the 11 studies included in this review.

The estimate of effect on SICH is more problematic to assess and has lowest power because of two separate definitions across studies and a small number of events. The CIs are also wide and cannot exclude either a small reduction or an increase in incidence of SICH. However, regardless of these two different definitions, there was no significant difference noted among the two groups

Systematic reviews of observational studies have the inherent weakness that in the absence of randomisation they are particularly prone to biases; for example, selection bias. It is not unreasonable to suppose that many (if not all) of the studies in the meta-analysis suffer from this kind of bias whereby only those elderly patients who seem particularly healthy or suitable are entered in the study cohort while those in the younger age group are selected less carefully. If this is true, then this will help to mask an increased risk of SICH for the older age group if it exists. The pooled analysis of appraised studies in this review shows case death ranging from $6.1 \%$ to $45.2 \%$ (mean $28.3 \%$; SD 12.0 ) in the thrombolysed $\geq 80$ year group. Given these data are from reasonably representative samples of hospital patients, there must be a considerable, but variable, amount of case selection (selection bias) in the included cohorts. For these reasons, the results provide no substitute for a randomised control trial. Moreover, if confounding bias is present such that there are one or more variables related to both age group and $\mathrm{SICH}$ which are not consequences of either, they can serve to distort the relationship between age group and SICH. The confounding variables which induce a negative association between age group and SICH are of particular interest since these will minimise any existing relationship between the variables. Some authors used logistic regression analysis to adjust for confounders, and this method is to be recommended. However, unadjusted-for confounders may still remain.

Publication bias may be an additional cause of bias whereby authors may be less willing to publish results which show a significant difference in SICH if it is against their a priori beliefs. However, the funnel plots did not suggest any evidence of publication bias for each of the outcomes in this meta-analysis.

Another potential bias is caused by loss to follow-up and/or protocol violations, especially differential loss to follow-up between the age groups. If those in the $<80$ year age group are followed-up less rigorously for example, and if loss to follow-up is mainly caused by greater health or mobility, then the proportion of patients in the $<80$ year age group associated with SICH or mortality will be biased upwards and this could contribute to a non-significant result. Unfortunately, only Parnetti $^{21}$ and Meseguer ${ }^{19}$ described and compared losses to follow-up.

In some studies, small numbers of elderly patients over 80 years of age may cause problems, not only because it suggests selection bias, but also because the sample size itself means that there may be low power to detect differences which are clinically significant. This means that the type II error rate would be high. This is also likely to be a feature of poorly designed and conducted studies. Therefore, in order to have any confidence in non-significant results it is especially important that studies are well conducted.

One important weakness which was not mentioned in any of the studies except Engelter ${ }^{16}$ is that of the disadvantage associated with using the dichotomisation of $<80$ years and $\geq 80$ years. The motivation for this is clear, as the NINDS study did not include many patients over 80 years, but it still seems unsatisfactory that an 80 year old patient should be treated any differently to a 79 year old. In reality, the relationship between age and outcome is likely to be gradual, as calculated by Berrouschot, ${ }^{14}$ and any dichotomisation of a continuous variable such as age is likely to result in a loss of power to detect a significant association. This is especially the case, for example, if probability of SICH increases only very slightly for patients 80-85 years and then increases more substantially after this. The problems associated with the dichotomisation provides another reason why an RCT in the elderly population is better than any comparison between two age groups $<80$ and $\geq 80$ years for tPA treated patients.

\section{CONCLUSION}

Elderly patients, thrombolysed for AIS, appear to have a lower probability of gaining favourable outcome and a higher mortality rate compared with younger patients less than 80 years of age. However, the rate of SICH was not significantly worse in the $\geq 80$ year olds compared with the younger patients.

These data support the rationale for recruiting patients aged over 80 years into ongoing trials comparing thrombolysis with control. For patients who refuse or cannot be randomised, this meta-analysis provides useful information on the potential risks and benefits of using alteplase off-licence.

\section{Competing interests None.}

Provenance and peer review Not commissioned; externally peer reviewed.

\section{REFERENCES}

1. Wardlow JM, Murray V, Berge E, et al. Thrombolysis for acute ischaemic stroke. Cochrane Database Syst Rev 2009;(4):CD000213. doi:10.1002/14651858.

2. SITS. Registration required for access. http://www.acutestroke.org/ laccessed 10 Sep 2010) 
3. Wardlow JM, Sandercock PA, Murray V. Should more patients with acute ischaemic stroke receive thrombolytic treatment? BMJ 2009;339:b4584.

4. Engelter ST, Bonati LH, Lyrer PA. Intravenous thrombolysis in stroke patients of $\geq 80$ versus $<80$ years of age - a systematic review across cohort studies. Age Ageing 2006;35:572-80.

5. Sweeting MJ, Sutton AJ, Lambert PC. What to add to nothing? Use and avoidance of continuity corrections in meta-analysis of spare data. Stat Med 2004;23:1351-75.

6. Egger M, Schneider M, Davey Smith G. Spurious precision? Meta-analysis of observational studies. BMJ 1998:316:140-4

7. Viechtbauer W. Metafor: meta-analysis package for R.R. package version 0.5-7 http://cran.r-project.org/package=metafor (accessed 10 Sep 2010).

8. R Development Core Team. R: A language and environment for statistical computing. R Foundation for Statistical Computing. Vienna, Austria. ISBN: 3-90005107-0. http://www.r-project.org/ (accessed 10 Sep 2010)

9. Chen $\mathbf{C l}$, Iguchi Y, Grotta JC, et al. Intravenous TPA for very old stroke patients. Eur Neurol 2005:54:140-4.

10. Tanne D, Gorman MJ, Bates VE, et al. Intravenous tissue plasminogen activator for acute ischaemic stroke in patients aged 80 years and older: the tPA stroke survey experience. Stroke 2000:31:370-5.

11. Mouradian MS, Senthilselvan A, Jickling G, et al. Intravenous rt-PA for acute stroke: comparing its effectiveness in younger and older patients. J Neurol Neurosurg Psychiatry 2005:76:1234-7.

12. Sylaja PN, Cote R, Buchan AM, et al; Canadian Alteplase for Stroke Effectiveness Study (CASES) Investigators. Thrombolysis in patients older than 80 years with acute ischaemic stroke: Canadian Alteplase for Stroke Effectiveness Study. J Neurol Neurosurg Psychiatry 2006;77:826-9.

13. Ringleb PA, Schwark C, Kohrmann M, et al. Thrombolytic therapy for acute ischaemic stroke in octogenarians: selection by magnetic resonance imaging improves safety but does not improve outcome. J Neurol Neurosurg Psychiatry 2007;78:690-3.

14. Berrouschot J, Rother J, Glahn Y, et al. Outcome and severe hemorrhagic complications of intravenous thrombolysis with tissue plasminogen activator in very old ( $\geq 80$ years) stroke patients. Stroke 2005;36:2421-5

15. Toni D, Lorenzano S, Agnelli G, et al. Intravenous thrombolysis with rt-PA in acute ischaemic stroke patients aged older than 80 years in Italy. Cerebrovasc Dis 2008;25:129-35

16. Engelter ST, Reichhart M, Sekoranja L, et al. Thrombolysis in stroke patients aged 80 years and older: Swiss survey of IV thrombolysis. Neurology 2005;65:1795-8.

17. Uyttenboogaart M, Schrijvers EM, Vroomen PC, et al. Routine thrombolysis with intravenous tissue plasminogen activator in acute ischaemic stroke patients aged 80 years or older: a single centre experience. Age Ageing 2007;36:577-9.

18. Gomez-Choco M, Obach V, Urra X, et al. The response to IV rt-PA in very old stroke patients. Eur J Neurol 2008;15:253-6.

19. Meseguer $\mathbf{E}$, Labreuche J, Olivot JM, et al. Determinants of outcome and safety of intravenous rt-PA therapy in the very old: a clinical registry study and systematic review. Age Ageing 2008;37:107-11.

20. van Oostenbrugge RJ, Hupperts RM, Lodder J. Thrombolysis for acute stroke with special emphasis on the very old: experience from a single Dutch centre. J Neurol Neurosurg Psychiatry 2006;77:375-7.

21. Parnetti L, Silvestrelli G, Lanari A, et al; Perugia Stroke and Neuroradiology Team. Efficacy of thrombolytic (rt-PA) therapy in old stroke patients: The Perugia stroke unit experience. Clin Exp Hypertens 2006;28:397-404.

22. The NINDS rt-PA Stroke Study Group. Tissue plasminogen activator for acute ischemic stroke. N Engl J Med 1995:333:1581-7.

23. Hacke W, Kaste M, Bluhmki E, et al. Thrombolysis with alteplase 3 to 4.5 hours after acute ischaemic stroke. N Engl J Med 2008;359:1317-29.

24. http://www.dcn.ed.ac.uk/ist3/ (accessed 10 Sep 2010).

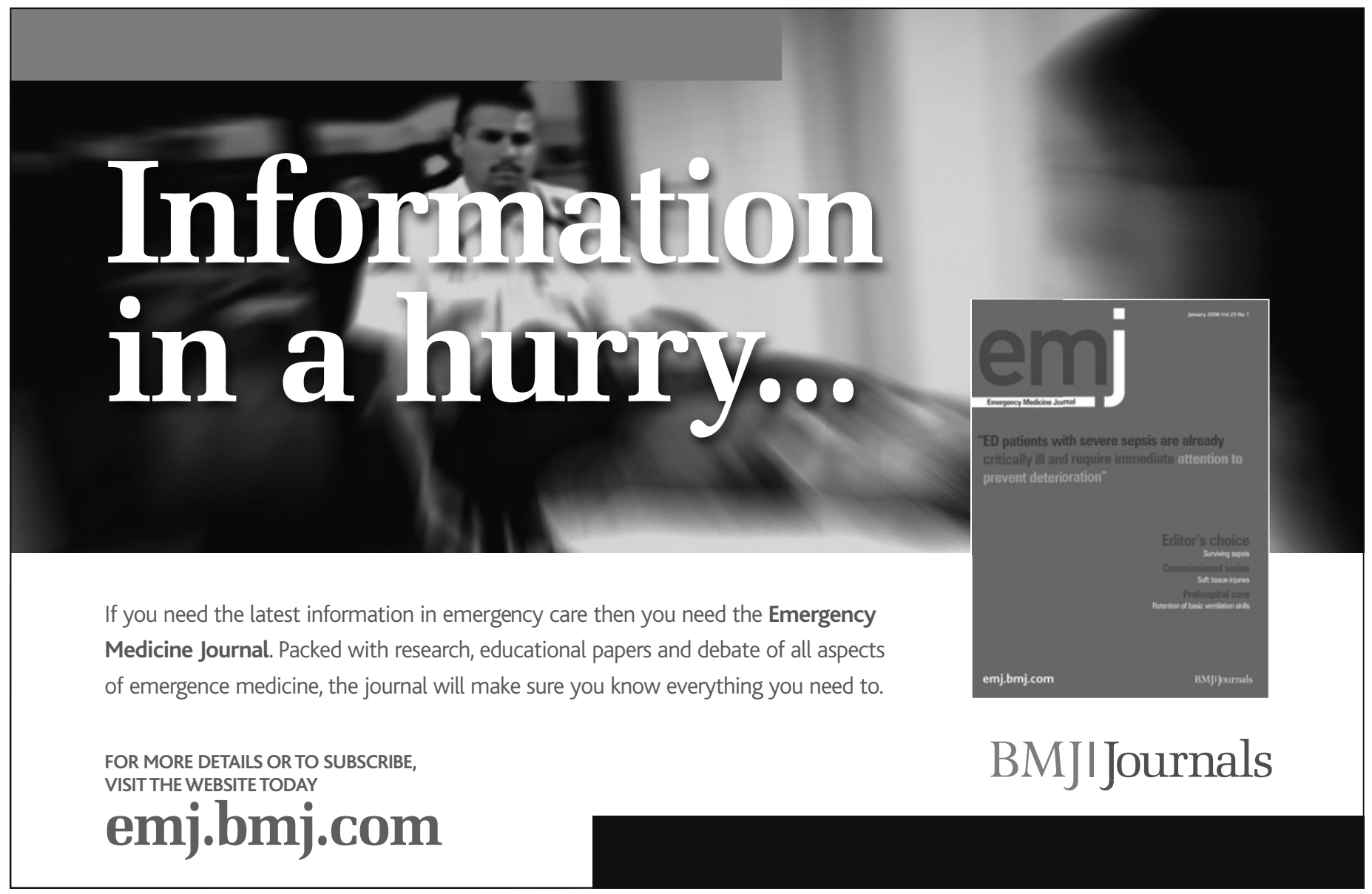

